\title{
To Existence of a Nonstationary Quasi-Linear Vector Field Realizing the Expansion of a Control Trajectory Bundle in Hilbert Space
}

\author{
VYACHESLAV RUSANOV \\ Department of Dynamics and Control \\ Matrosov Institute for System Dynamics and Control Theory \\ of the Siberian Branch of the Russian Academy of Sciences \\ st. Lermontova, 134, Irkutsk \\ RUSSIA \\ v.rusanov@mail.ru \\ ALEKSEY DANEEV \\ Department of Information Systems \\ Irkutsk State Transport University \\ st. Chertyshevsky, 15, Irkutsk \\ RUSSIA \\ daneev@mail.ru \\ ANATOLIY LAKEYEV \\ Department of Dynamics and Control \\ Matrosov Institute for System Dynamics and Control Theory \\ of the Siberian Branch of the Russian Academy of Sciences \\ st. Lermontova, 134, Irkutsk \\ RUSSIA \\ lakeyev@icc.ru \\ YURIJ LINKE \\ Department of Applied Mathematics and Information Science \\ Irkutsk National Research Technical University \\ st. Lermontova, 83, Irkutsk \\ RUSSIA \\ linkeyurij@gmail.com
}

Abstract: - The study of algebraic extension of a countable family of controlled non-linear dynamic processes "input-output" having differential realization in the class of ordinary quasi-linear differential equations (with software-positional control and without) in a separable Hilbert space was conducted. This problem as a starting point for the development of the general theory of vector fields, simultaneously creating a reputation for it as a useful tool in precision mathematical modeling of complex dynamic systems.

Key-Words: - non-linear dynamic processes, non-linear differential realization, non-stationary $\left(A, B, B^{\#}\right)_{2}$-model of vector field.

Received: February 25, 2020. Revised: April 30, 2020. Accepted: May 15, 2020. Published: May 29, 2020.

\section{Introduction}

In this paper we introduce and study (on the qualitative level) the concept of elementary (singleton) expansion of a countable bundle of non-linear dynamic processes allowing differential implementation in the class of quasi-linear controlled systems in the infinite-dimensional Hilbert space. The re- ceived results are a natural evolution of non-linear system analysis of complex differential models [1-3] and may be useful in the study of inverse problems for partial differential equations [4, 5]; statement of the problem considered below, was proposed in the conclusions of the work [3]. 


\section{Statement of the Problem}

Further $\left(X,\|\cdot\|_{X}\right),\left(Y,\|\cdot\|_{Y}\right),\left(Z,\|\cdot\|_{Z}\right)$ are real separable Hilbert spaces (pre-Hilbert [6] define norms $\|\cdot\|_{X}$, $\left.\|\cdot\|_{Y},\|\cdot\|_{Z}\right), U:=X \times Y \times Z$ is the Hilbert space with the norm $\|(\mathrm{x}, \mathrm{y}, \mathrm{z})\|_{U}:=\left(\|\mathrm{x}\|_{X}^{2}+\|\mathrm{y}\|_{Y}^{2}+\|\mathrm{z}\|_{Z}^{2}\right)^{1 / 2}, \mathrm{x}, \mathrm{y}, \mathrm{z} \in$ $U, L(Y, X)$ is the Banach space with the operator norm $\|\cdot\|_{L(Y, X)}$ of all linear continuous operators from the space $Y$ to the space $X\left(\operatorname{similar}\left(L(X, X),\|\cdot\|_{L(X, X)}\right)\right.$ and $\left.\left(L(Z, X),\|\cdot\|_{L(Z, X)}\right)\right), T:=\left[t_{0}, t_{1}\right]$ is a segment of the real line $R$ with the Lebesgue measure $\mu$ and $\wp_{\mu}$ is the $\sigma$-algebra of all $\mu$-measurable subsets of the interval $T$.

If below $(\mathscr{B},\|\cdot\|)$ is a some Banach space, then as usual through $\mathrm{L}_{2}(T, \mu, \widetilde{B})$ we will denote Banach quotient-space of classes $\mu$-equivalence of all integrable maps $f: T \rightarrow \mathscr{B}$ of Bochner [6, c. 189] with the norm $\left(\int_{T}\|f(\tau)\|^{2} \mu(d \tau)\right)^{1 / 2}$. In addition everywhere further $A C(T, X)$ is the linear set of all absolutely continuous on $T$ functions (with respect to $\mu$ measure) with values in the space $X$, moreover

$$
\begin{gathered}
\boldsymbol{\Pi}:=A C(T, X) \times \mathrm{L}_{2}(T, \mu, Y) \times \mathrm{L}_{2}(T, \mu, Z), \\
\mathbf{L}_{2}:=\mathrm{L}_{2}(T, \mu, L(X, X)) \times \\
\times \mathrm{L}_{2}(T, \mu, L(Y, X)) \times \mathrm{L}_{2}(T, \mu, L(Z, X)) .
\end{gathered}
$$

Now we will distinguish for consideration controlled differential models of the form

$$
d x / d t=A x+B u+B^{\#} u^{\#}(x),
$$

where $\left(x, u, u^{\sharp}(x)\right) \in \Pi, x$ is a Carathéodory solution ${ }^{1}$ ( $C$-solution), $u$ is a programmed control, $u^{\#}(x)$ is a poly-linear control (state feedback), and $\left(A, B, B^{\#}\right) \in$ $\mathbf{L}_{2}$; in purposes of terminological convenience triple of vector-functions $\left(x, u, u^{\#}(x)\right)$ we will also call $C$ solution and triple of operator-functions $\left(A, B, B^{\#}\right)$, adhering the terminology from [7-8], we will call non-stationary $\left(A, B, B^{\#}\right)_{2}$-model of vector field [5] of differential equation (1).

The problem of elementary extension of quasi-linear differential realization of the bundle of dynamic processes [7]: for a given poly-linear law

$$
x \mapsto u^{\#}(x): A C(T, X) \rightarrow \mathrm{L}_{2}(T, \mu, Z)
$$

and fixed families $N, N^{*}$ of processes "input-output" such that

$$
\begin{gathered}
N, N^{*} \subset\left\{(x, u, q) \in \boldsymbol{\Pi}:(x, u, q)=\left(x, u, u^{\sharp}(x)\right)\right\}, \\
1 \leq \text { Card } N \leq \boldsymbol{N}_{0} \text { (aleph-zero), } \\
\text { Card } N^{*}=1, N^{*} \not \subset N,
\end{gathered}
$$

where $N, N^{*}$ have individual differential realizations (1), to determine analytical conditions under which $N \cup N^{*}$ is a family of $C$-solutions of some differential equation (1).

\footnotetext{
${ }^{1}$ When equality in (1) is considered as identity in Banach space $\mathrm{L}_{1}(T, \mu, X)$.
}

\section{Existence of Elementary Extension of Countable Quasi-Linear Bundle of Control Dynamic Processes}

We endow the space

$$
H_{2}:=\mathrm{L}_{2}(T, \mu, X) \times \mathrm{L}_{2}(T, \mu, Y) \times \mathrm{L}_{2}(T, \mu, Z)
$$

with the topology of the norm

$$
\left(\int_{T}\|(g(\tau), w(\tau), q(\tau))\|_{U}^{2} \mu(d \tau)\right)^{1 / 2}, \quad(g, w, q) \in H_{2} ;
$$

clear, that $\mathrm{H}_{2}$ is the Hilbert space [6].

We differ the element $\left(x, u, u^{\sharp}(x)\right) \in \boldsymbol{\Pi}$ in the notations as class of equivalence (i.e. element out of the space $\mathrm{H}_{2}$ ) from the specific representative (vectorfunction) $\left(x(\cdot), u(\cdot), u^{\#}(x(\cdot))\right)$ from this class.

We will denote through $G_{E}$ arbitrary (but fixed and numbered) algebraic basis in $E:=$ Span $N$ and let $\left\{\left(x^{*}, u^{*}, u^{\#}\left(x^{*}\right)\right)\right\}:=N^{*}$, while $\left(x^{*}, u^{*}, u^{\#}\left(x^{*}\right)\right) \notin E$. It is obvious that at any point $t \in T$ expansion in the Hilbert space of $U$ vector $\left(x^{*}(t), u^{*}(t), u^{\#}\left(x^{*}(t)\right)\right)$ is possible on the projection in

$$
\begin{aligned}
& \operatorname{Span}\left\{\left(x(t), u(t), u^{\#}(x(t))\right)\right)_{i}: \\
& \left.\left(x(\cdot), u(\cdot), u^{\#}(x(\cdot))\right)_{i} \in G_{E}, i=1,2, \ldots\right\} \subset U,
\end{aligned}
$$

which is denoted by $\left(x_{-}^{*}(t), u_{-}^{*}(t), u_{-}^{\#}\left(x^{*}(t)\right)\right)$ and addition

$$
\begin{gathered}
\left(x_{\perp}^{*}(t), u_{\perp}^{*}(t), u_{{ }_{\perp}}\left(x^{*}(t)\right)\right):= \\
=\left(x^{*}(t), u^{*}(t), u^{\#}\left(x^{*}(t)\right)\right)-\left(x_{-}^{*}(t), u_{-}^{*}(t), u_{-}^{\#}\left(x^{*}(t)\right)\right) .
\end{gathered}
$$

Lemma 1. Vector-functions

$$
\begin{aligned}
t & \mapsto\left(x^{*}(t), u_{-}^{*}(t), u_{-}^{\#}\left(x^{*}(t)\right)\right): T \rightarrow U, \\
t & \mapsto\left(x^{*}{ }_{\perp}(t), u_{\perp}^{*}(t), u_{\perp}^{\#}\left(x^{*}(t)\right)\right): T \rightarrow U
\end{aligned}
$$

are $\mu$-measurable mappings.

(By the separability of $U$ weak and strong measurabilities coincide [6, p. 187].)

Lemma 2. Representation

$$
\begin{gathered}
\left(x^{*}, u^{*}, u^{\#}\left(x^{*}\right)\right)= \\
=\left(x^{*}, u_{-}^{*}, u_{-}^{\#}\left(x^{*}\right)\right)+\left(x_{\perp}^{*}, u_{\perp}^{*}, u^{\#}{ }_{\perp}\left(x^{*}\right)\right)
\end{gathered}
$$

doesn't depend on the choice of algebraic basis $G_{E}$, while

$$
\left(x_{-}^{*}, u_{-}^{*}, u_{-}^{\#}\left(x^{*}\right)\right),\left(x^{*}{ }_{\perp}, u_{\perp}^{*}, u_{\perp}^{\#}\left(x^{*}\right)\right) \in H_{2} \text {. }
$$

We denote through $\Omega_{E}$ and $\Omega^{*}{ }_{\perp}$ circuits in the space $\mathrm{H}_{2}$ respectively to linear manifolds $\operatorname{Span}\{\chi \cdot(x$, $\left.\left.u, u^{\#}(x)\right): \chi \in F,\left(x, u, u^{\sharp}(x)\right) \in E\right\}$ and $\operatorname{Span}\left\{\chi \cdot\left(x^{*}, u^{*}{ }_{\perp}\right.\right.$, $\left.\left.u_{\perp}^{\#}\left(x^{*}\right)\right): \chi \in F\right\}$, where $F$ is a family of equivalence classes $(\bmod \mu)$ of all characteristic functions induced by elements of $\sigma$-algebra $\wp_{\mu}$.

Lemma 3. Subspaces $\Omega_{E}, \Omega^{*}{ }_{\perp} \subset H_{2}$ are orthogonal, i.e. $\Omega_{E} \perp \Omega^{*}$.

Remark 1. Everywhere further for two closed subspaces from the space $\mathrm{H}_{2}$, such that their intersection is $\{0\} \subset H_{2}$, and the vector sum is closed in $\mathrm{H}_{2}$ we agree to denote the sign of their vector addition through $\oplus$, in particular, Theorem 14.C [9, p. 42] and Lemma 3 make note $\Omega_{E} \oplus \Omega^{*}{ }_{\perp}$ correctly. 
We ask the question: what are the analytical conditions imposed on the sets of controlled dynamic processes $N$ and $\left\{\left(x^{*}, u^{*}, u^{\#}\left(x^{*}\right)\right)\right\}$, "extended" family of processes $N \cup\left\{\left(x^{*}, u^{*}, u^{\#}\left(x^{*}\right)\right)\right\}$ has a differential realization (1)? On one of the ways of geometric solution of this problem is construction of characteristic feature (see below Theorem 1) defining equality

$$
\Omega_{E}+\Omega^{*}=\Omega_{E} \oplus \Omega_{\perp}^{*}
$$

where $\Omega^{*}$ is closure in the space $H_{2}$ of linear manifold $\operatorname{Span}\left\{\chi \cdot\left(x^{*}, u^{*}, u^{\#}\left(x^{*}\right)\right): \chi \in F\right\}$, while a particular form of equation (2), namely, of the type

$$
\Omega_{E} \oplus \Omega^{*}=\Omega_{E} \oplus \Omega^{*} \text {, }
$$

positively responds to the aforesaid issue about the realization of the expanded bundle $N \cup\left\{\left(x^{*}, u^{*}\right.\right.$, $\left.\left.u^{\sharp}\left(x^{*}\right)\right)\right\}$ in the context of approach to geometric solution of the task of expansion of differential realization based on the Theorem 14.C [9, p. 42] and Theorem 3 [7]; below Theorem 2 detects one characteristic property of equality (3).

Further $T_{0}:=\left\{t \in T:\left(x^{*}{ }_{\perp}(t), u^{*} \perp(t), u^{\#} \perp\left(x^{*}(t)\right)\right)=0\right\}$ $\in \wp_{\mu}, v^{*}{ }_{\perp}, v^{*}$ are Lebesgue replenishments of measures

$$
\begin{gathered}
\int_{S}\left\|\left(x^{*}{ }_{\perp}(\tau), u^{*}{ }_{\perp}(\tau), u^{\#}\left(x^{*}(\tau)\right)\right)\right\|_{U}^{2} \mu(d \tau), S \in \wp_{\mu}, \\
\int_{S}\left\|\left(x^{*}(\tau), u^{*}(\tau), u^{\#}\left(x^{*}(\tau)\right)\right)\right\|_{U}^{2} \mu(d \tau), S \in \wp_{\mu} .
\end{gathered}
$$

Theorem 1. $\Omega_{E}+\Omega^{*}=\Omega_{E} \oplus \Omega^{*}{ }_{\perp}$ only if

$$
\mathrm{L}_{2}\left(T, v^{*}, R\right)=\chi_{\perp} \cdot \mathrm{L}_{2}\left(T, v^{*}, R\right) \text {, }
$$

where $\chi_{\perp} \in F$ is the characteristic function of the set $T \mid T_{0}$.

Proof of Theorem 1 we reduce to the establishment of Lemma 4 and Lemma 5.

Lemma 4. $\Omega_{E}+\Omega^{*} \subset \Omega_{E} \oplus \Omega^{*}$.

Proof. Let $\omega^{\prime} \in \Omega^{*}$, then according to Lemma 4 [7] will be

$$
\begin{gathered}
\omega^{\prime}=\lambda^{\prime} \cdot\left(x^{*}, u^{*}, u^{\#}\left(x^{*}\right)\right)= \\
=\lambda^{\prime} \cdot\left(x^{*}, u^{*}, u^{*}\left(x^{*}\right)\right)+\lambda^{\prime} \cdot\left(x^{*}{ }_{\perp}, u_{\perp}{ }_{\perp}, u^{\#}{ }_{\perp}\left(x^{*}\right)\right),
\end{gathered}
$$

where $\lambda^{\prime} \in \mathrm{L}_{2}\left(T, v^{*}, R\right)$. Further, since for each function $\lambda \in \mathrm{L}_{2}\left(T, v^{*}, R\right)$ we have

$$
\begin{gathered}
\lambda^{2}(t)\left\|\left(x^{*}(t), u^{*}(t), u^{\#}\left(x^{*}(t)\right)\right)\right\|_{U}^{2} \geq \\
\geq \lambda^{2}(t)\left\|\left(x^{*}{ }_{\perp}(t), u^{*}{ }_{\perp}(t), u^{\#}{ }_{\perp}\left(x^{*}(t)\right)\right)\right\|_{U}^{2},
\end{gathered}
$$

then the following embedding of functional spaces is true

$$
\mathrm{L}_{2}\left(T, v^{*}, R\right) \subset \mathrm{L}_{2}\left(T, v^{*}{ }_{\perp}, R\right),
$$

where $\lambda^{\prime} \cdot\left(x^{*}{ }_{\perp}, u^{*}{ }_{\perp}, u^{\#}{ }_{\perp}\left(x^{*}\right)\right) \in \Omega^{*}{ }_{\perp}$ (based on the analytical structure of the subspace $\Omega^{*}$, given in Lemma 4 [7]). Thus, by the arbitrariness of the choice of the element $\omega^{\prime} \in \Omega^{*}$, the lemma will be proved as soon as we discover:

$$
\lambda^{\prime} \cdot\left(x^{*}, u_{-}{ }_{-}, u_{-}^{\#}\left(x^{*}\right)\right) \in \Omega_{E} .
$$

For this it is sufficient to show (Corollary [6, p. 157]) that $<\lambda^{\prime} \cdot\left(x_{-}^{*}, u_{-}{ }_{-}, u_{-}^{\#}\left(x^{*}\right)\right), \omega^{\prime \prime}>_{H_{2}}=0$, where $<\cdot, \cdot\rangle_{H_{2}}$ is the scalar product in $H_{2}$, for all $\omega^{\prime \prime} \in H_{2}$, such that $\left\langle\omega^{\prime \prime}, \omega\right\rangle_{H_{2}}=0$, for any $\omega \in \operatorname{Span}\{\chi \cdot(x, u$, $\left.\left.u^{\sharp}(x)\right): \chi \in F,\left(x, u, u^{\sharp}(x)\right) \in E\right\}$, which is equivalent to install:

$$
\begin{gathered}
\omega^{\prime \prime}(t) \perp \operatorname{Span}\left\{\left(x(t), u(t), u^{\#}(x(t))\right)_{i}:\right. \\
\left.\left(x(\cdot), u(\cdot), u^{\#}(x(\cdot))\right)_{i} \in G_{E}, i=1,2, \ldots\right\}
\end{gathered}
$$

$\mu$-almost everywhere in $T$, here $\perp$ is the relation of orthogonality in the space $U$.

We expand vector-function $\omega^{\prime \prime}(\cdot)$ in each point $t \in$ $T$ in the sum of

Where

$$
\omega^{\prime \prime}(t)+\omega^{\prime \prime}{ }_{\perp}(t):=\omega^{\prime \prime}(t),
$$

$$
\omega^{\prime \prime} \_(t) \in \operatorname{Span}\left\{\left(x(t), u(t), u^{\#}(x(t))\right) i:\right.
$$

$\left.\left(x(\cdot), u(\cdot), u^{\sharp}(x(\cdot))\right)_{i} \in G_{E}, i=1,2, \ldots\right\}$,

and $\omega^{\prime \prime} \perp_{\perp}(t)$ is orthogonal to

$$
\operatorname{Span}\left\{\left(x(t), u(t), u^{\#}(x(t))\right)_{i}\right. \text { : }
$$

$$
\left.\left(x(\cdot), u(\cdot), u^{\#}(x(\cdot))\right)_{i} \in G_{E}, i=1,2, \ldots\right\} .
$$

Then if $\omega^{\prime \prime} \neq 0$, there exists such set $S^{*} \in \wp_{\mu}$, $\mu\left(S^{*}\right)>0$, that $\omega^{\prime \prime}(t) \neq 0, \forall t \in S^{*}$, while in the basis $G_{E}$ there is such vector $\left(x(\cdot), u(\cdot), u^{\#}(x(\cdot))\right)$, that is

$$
\left(x(t), u(t), u^{\sharp}(x(t))\right)_{i} \neq 0
$$

$\mu$-almost everywhere in set $S^{*}$; otherwise for $\mu$-almost all $t \in S^{*}$ equalities will be "realized"

$$
\operatorname{Span}\left\{\left(x(t), u(t), u^{\sharp}(x(t))\right)_{i}\right. \text { : }
$$

$\left.\left(x(\cdot), u(\cdot), u^{\#}(x(\cdot))\right)_{i} \in G_{E}, i=1,2, \ldots\right\}=\{0\} \subset U$,

and therefore $\omega^{\prime \prime}=0$ should be performed in this position.

Now we denote through $S^{*}$ and $S^{*}$ _ subsets (partition) $S^{*}$ equal

$$
\begin{aligned}
& S^{*}{ }_{+}=\left\{t \in S^{*}:<\omega^{\prime \prime}(t),\left(x(t), u(t), u^{\#}(x(t))\right)_{i}>_{U} \geq 0\right\} \in \wp_{\mu}, \\
& S_{-}^{*}=\left\{t \in S^{*}:<\omega^{\prime \prime}(t),\left(x(t), u(t), u^{\#}(x(t))\right)_{i}>_{U}<0\right\} \in \wp_{\mu} .
\end{aligned}
$$

It is obvious that at least one of the sets $S^{*}{ }_{+}$or $S^{*}$ has a nonzero measure. Let $S^{*}{ }_{+}$acts as such set. Then

$$
\begin{gathered}
\chi+\cdot\left(x, u, u^{\sharp}(x)\right)_{i} \in \operatorname{Span}\left\{\chi \cdot\left(x, u, u^{\sharp}(x)\right):\right. \\
\left.\chi \in F,\left(x, u, u^{\sharp}(x)\right) \in E\right\}
\end{gathered}
$$

and $\left\langle\omega^{\prime \prime}, \chi_{+} \cdot\left(x, u, u^{\#}(x)\right)_{i}>_{H_{2}}>0\right.$, where $\chi_{+}$is the characteristic function of a set $S^{*}$. It is clear that we obtain $<\omega^{\prime \prime}, \chi+\cdot\left(x, u, u^{\#}(x)\right)_{i}>_{H_{2}}>0$ whereby we arrive at a contradiction with the conditions defined above the construction of the functional $\omega^{\prime \prime}$.

The above proof provides a useful clarification:

Corollary 1. $\mathrm{L}_{2}\left(T, v^{*}, R\right) \subset \mathrm{L}_{2}\left(T, v^{*}{ }_{\perp}, R\right)$.

Lemma 5. $\Omega_{E}+\Omega^{*} \supset \Omega_{E} \oplus \Omega_{\perp}{ }_{\perp} \Leftrightarrow \mathrm{L}_{2}\left(T, v^{*}, R\right)=$ $\chi_{\perp} \cdot \mathrm{L}_{2}\left(T, v^{*}, R\right)$.

Proof. $(\Rightarrow)$. Let $\lambda_{\omega} \in \mathrm{L}_{2}\left(T, v^{*}{ }_{\perp}, R\right)$ and $\omega:=\lambda_{\omega} \cdot\left(x^{*}{ }_{\perp}\right.$, $u_{\perp}^{*}, u_{\perp}{ }_{\perp}\left(x^{*}\right)$ ), where (Lemma 4 [7]) $\omega \in \Omega_{E} \oplus \Omega^{*}{ }_{\perp}$, means (assumption $\Rightarrow$ ) $\omega \in \Omega_{E}+\Omega^{*}$. Then by $\omega \in \Omega_{E}$ $\oplus \Omega^{*}{ }_{\perp}$ vector $\omega$ has an expansion of (unique) form

$$
\omega=\omega^{\prime}+\lambda_{\omega} \cdot\left(x^{*}{ }_{\perp}, u^{*} \perp, u^{\#}{ }_{\perp}\left(x^{*}\right)\right),
$$

where $\omega^{\prime}=0 \in \Omega_{E}$, at this effect $\omega \in \Omega_{E}+\Omega^{*}$ representation is true:

$$
\omega=\omega^{\prime \prime}+\lambda^{*} \cdot\left(x^{*}, u^{*}, u^{\#}\left(x^{*}\right)\right)=
$$


$=\omega^{\prime \prime}+\lambda^{*} \cdot\left(x^{*}{ }_{,}, u^{*}{ }_{-}, u_{-}^{\#}\left(x^{*}\right)\right)+\lambda^{*} \cdot\left(x^{*}{ }_{\perp}, u_{\perp}{ }_{\perp}, u^{\#} \perp\left(x^{*}\right)\right)$, where $\omega^{\prime \prime} \in \Omega_{E}, \lambda^{*} \in \mathrm{L}_{2}\left(T, v^{*}, R\right)$. Since (reasoning's are similar to the proof of Lemma 4 [7]) the inclusions take place $\lambda^{*} \cdot\left(x_{-}{ }_{-}, u_{-}{ }_{-}, u_{-}^{\#}\left(x^{*}\right)\right) \in \Omega_{E}, \lambda^{*} \cdot\left(x^{*}{ }_{\perp}, u^{*}{ }_{\perp}\right.$, $\left.u_{\perp}^{\#}\left(x^{*}\right)\right) \in \Omega^{*}{ }_{\perp}$, thet $\omega^{\prime}=\omega^{\prime \prime}+\lambda^{*} \cdot\left(x^{*}{ }_{-}, u^{*}{ }_{-}, u^{\#}\left(x^{*}\right)\right)$ and $\lambda_{\omega} \cdot\left(x^{*} \perp, u^{*} \perp, u^{\#} \perp\left(x^{*}\right)\right)=\lambda^{*} \cdot\left(x^{*} \perp, u^{*}, u^{\#} \perp\left(x^{*}\right)\right)$. Thus, taking into account the presence of a linear isometry between $\mathrm{L}_{2}\left(T, v^{*}{ }_{\perp}, R\right)$ and $\Omega^{*}{ }_{\perp}$ (Lemma 4 [7]) will be $\lambda_{\omega}$ $=\chi_{\perp} \cdot \lambda^{*}$, where in the end by the arbitrariness of the choice of function $\lambda_{\omega}$, we obtain

$$
\mathrm{L}_{2}\left(T, v^{*}{ }_{\perp}, R\right) \subset \chi_{\perp} \cdot \mathrm{L}_{2}\left(T, v^{*}, R\right)
$$

or, taking into account Corollary 1 , will be

$$
\mathrm{L}_{2}\left(T, v^{*}{ }_{\perp}, R\right)=\chi_{\perp} \cdot \mathrm{L}_{2}\left(T, v^{*}, R\right) \text {. }
$$

$(\Leftarrow)$. Let $\omega \in \Omega_{E} \oplus \Omega_{\perp}^{*}$. Then $\omega=\omega^{\prime}+\lambda_{\omega} \cdot\left(x^{*} \perp\right.$, $\left.u^{*}{ }_{\perp}, u^{\#}{ }_{\perp}\left(x^{*}\right)\right)$, where $\omega^{\prime} \in \Omega_{E}, \lambda_{\omega} \in \mathrm{L}_{2}\left(T, v^{*}{ }_{\perp}, R\right)$. Since (assumption $\Leftarrow) \lambda_{\omega} \in \chi_{\perp} \mathrm{L}_{2}\left(T, v^{*}, R\right)$, then we have a bunch of equalities

$$
\begin{gathered}
\omega^{\prime}+\lambda_{\omega} \cdot\left(x^{*}{ }_{\perp}, u_{\perp}{ }_{\perp}, u_{\perp}{ }_{\perp}\left(x^{*}\right)\right)= \\
=\omega^{\prime}+\lambda_{\omega} \cdot\left(x^{*}{ }_{\perp}, u^{*}{ }_{\perp}, u^{\#}{ }_{\perp}\left(x^{*}\right)\right)+ \\
+\lambda_{\omega} \cdot\left(x^{*}, u_{-} u^{*}, u^{\#}\left(x^{*}\right)\right)-\lambda_{\omega} \cdot\left(x^{*}, u^{*}, u^{\#}\left(x^{*}\right)\right)= \\
=\omega^{\prime}-\lambda_{\omega} \cdot\left(x^{*}, u^{*}{ }_{-}, u^{\#}\left(x^{*}\right)\right)+\lambda_{\omega} \cdot\left(x^{*}, u^{*}, u^{\#}\left(x^{*}\right)\right),
\end{gathered}
$$

therefore, $\omega \in \Omega_{E}+\Omega^{*}$ taking into account

$$
\begin{gathered}
\left(\omega^{\prime}-\lambda_{\omega} \cdot\left(x^{*}, u_{,}^{*}, u_{-}^{\#}\left(x^{*}\right)\right)\right) \in \Omega_{E}, \\
\lambda_{\omega} \cdot\left(x^{*}, u^{*}, u^{\#}\left(x^{*}\right)\right) \in \Omega^{*} .
\end{gathered}
$$

Now we present a variant of characteristic conditions of equality (3).

Theorem 2. If we implement $T_{0}=\varnothing(\bmod \mu)$ offer is valid:

Proof. That is

$$
\begin{gathered}
\Omega_{E} \oplus \Omega^{*}=\Omega_{E} \oplus \Omega^{*}{ }_{\perp} \Leftrightarrow \\
\Leftrightarrow \mathrm{L}_{2}\left(T, v^{*}{ }^{*}, R\right)=\mathrm{L}_{2}\left(T, v^{*}, R\right) .
\end{gathered}
$$

$$
\begin{aligned}
& \Omega_{E}+\Omega^{*}=\Omega_{E} \oplus \Omega^{*}{ }_{\perp} \Leftrightarrow \\
\Leftrightarrow & \mathrm{L}_{2}\left(T, v^{*}{ }_{\perp}, R\right)=\mathrm{L}_{2}\left(T, v^{*}, R\right)
\end{aligned}
$$

is a direct statement of Theorem 1 . On the other hand, confirmation of equality

follows from the assumption

$$
\Omega_{E} \cap \Omega^{*}=\{0\} \subset H_{2}
$$

$$
\left\{t \in T:\left(x^{*}{ }_{\perp}(t), u^{*}{ }_{\perp}(t), u^{\#}{ }_{\perp}\left(x^{*}(t)\right)\right)=0\right\}=\varnothing(\bmod \mu)
$$

and Corollary of Mazur's Theorem [6, p. 157].

Theorem 1 (given the finding of Lemma 5) and Theorem 2 attracting Theorem 14.C from [9, p. 42] and Theorem 3 from [7] do a fair conclusion:

Corollary 2. The following three properties are equivalent:

$$
\begin{gathered}
\mathrm{L}_{2}\left(T, v^{*}{ }_{\perp}, R\right) \subset \chi_{\perp} \cdot \mathrm{L}_{2}\left(T, v^{*}, R\right) \Leftrightarrow \\
\Leftrightarrow \mathrm{L}_{2}\left(T, v^{*}{ }^{*}, R\right)=\chi_{\perp} \cdot \mathrm{L}_{2}\left(T, v^{*}, R\right) \Leftrightarrow \\
\Leftrightarrow \Omega_{E} \oplus \Omega^{*}{ }_{\perp}=\Omega_{E}+\Omega^{*},
\end{gathered}
$$

and if $T_{0}=\varnothing(\bmod \mu)$, then any signified property turns the bundle $N \cup\left\{\left(x^{*}, u^{*}, u^{\#}\left(x^{*}\right)\right)\right\}$ into the set of dynamic processes with the quasi-linear differential realization (1).

Remark 2. Corollary 2 allows to call Theorem 2 as "direct theorem" about elementary algebraic ex- tension of differential realization while hypothesis: $T_{0}=\varnothing(\bmod \mu), N \cup\left\{\left(x^{*}, u^{*}, u^{\#}\left(x^{*}\right)\right)\right\}$ has a realization $(1) \Rightarrow \mathrm{L}_{2}\left(T, v^{*}{ }_{\perp}, R\right) \subset \mathrm{L}_{2}\left(T, v^{*}, R\right)$ in general case isn't confirmed that the following example illustrates.

Example 1. Let $X=Y=R, T=[-1,1], u^{\#}(\cdot)=0$ and

$$
\begin{gathered}
N=\left\{t \mapsto\left(\mathrm{e}^{-t}, 0,0\right): t \in T\right\}, \\
\left\{\left(x^{*}, u^{*}, u^{\#}\left(x^{*}\right)\right)\right\}=\left\{t \mapsto\left(\mathrm{e}^{-t}+t-1, t, 0\right): t \in T\right\} ;
\end{gathered}
$$

it is obvious that $T_{0}=\varnothing(\bmod \mu)$ and the bundle $N$ $\cup\left\{\left(x^{*}, u^{*}, u^{\#}\left(x^{*}\right)\right)\right\}$ has a realization $(1)$; we note that $T_{0} \neq \varnothing$. Then $\mathrm{L}_{2}\left(T, v^{*}, R\right)=\mathrm{L}_{2}(T, \mu, R)$ and $\mathrm{L}_{2}\left(T, v^{*}{ }_{\perp}, R\right)$, $v^{*}{ }_{\perp}=\int \tau^{2} \mu(d \tau)$, because $\left(x^{*}{ }_{\perp}(t), u^{*}{ }_{\perp}(t), u^{\#} \perp\left(x^{*}(t)\right)\right)=(0$, $t, 0)$. It is clear that $1 / t \in \mathrm{L}_{2}\left(T, v^{*}, R\right), 1 / t \notin \mathrm{L}_{2}(T, \mu, R)$, where $\mathrm{L}_{2}\left(T, v^{*}{ }_{\perp}, R\right) \not \subset \mathrm{L}_{2}\left(T, v^{*}, R\right)$; hence by Lemma 5 we also conclude that

$$
\Omega_{E} \oplus \Omega^{*}{ }_{\perp} \not \subset \Omega_{E}+\Omega^{*} .
$$

Next statement shows that the construction similar to Example 1 can't be realized in the functional class $A C(T, X) \times\{0\} \times\{0\} \subset \boldsymbol{\Pi}$, i.e. for free trajectories ( $C$-solutions) it can be said that for $N \subset A C(T, X)$ $\times\{0\} \times\{0\}$ Corollary 3 in a known sense is opposite to Corollary 2 (see above Remark 2 ).

Corollary 3. If $N \subset A C(T, X) \times\{0\} \times\{0\} \subset \boldsymbol{\Pi}$, Card $N<\infty$ and $N \cup\left\{\left(x^{*}, 0,0\right)\right\} \subset \Pi$ is a set of trajectories with the differential realization (1) with

$$
\begin{gathered}
u(\cdot)=0 \in \mathrm{L}_{2}(T, \mu, Y), \\
u^{\#}(\cdot)=0 \in \mathrm{L}_{2}(T, \mu, Z),
\end{gathered}
$$

then the following relations are true:

$$
\begin{gathered}
T_{0}=\varnothing, \\
\mathrm{L}_{2}\left(T, v^{*}{ }_{\perp}, R\right)=\mathrm{L}_{2}\left(T, v^{*}, R\right), \\
\Omega_{E} \oplus \Omega^{*}=\Omega_{E} \oplus \Omega^{*}{ }_{\perp} .
\end{gathered}
$$

Proof. It is easy to see that $T_{0}=\varnothing$, because otherwise there exists a period of time $t_{*} \in T$, which $x^{*}(t *)=\Sigma \alpha_{i} x_{(i)}\left(t_{*}\right)$, where all constants $\alpha_{i}$, except the finite number are zero, $x_{(i)}$ is the first component of the triple $(x, 0,0)_{i} \in G_{E}$. Consequently, the trajectory $x^{*}(\cdot)$ has a representation $\Sigma \alpha_{i} x_{(i)}(\cdot)$ by the uniqueness of solution, extending at time $t *$ through the point $x^{*}(t *)$, for the differential system (1) with $(A, 0,0)_{2}$ model, corresponding to a set of dynamic processes $N \cup\left\{\left(x^{*}, 0,0\right)\right\}$; that is contrary to its earlier condition $\left(x^{*}, 0,0\right) \notin E$.

Further, because of the continuity of the trajectory $x^{*}(\cdot)$ and the compactness of the interval $T$, there exist such real constants $c_{1}, c_{2}>0$, that equalities are true

$$
\begin{gathered}
\inf \left\{\left\|x^{*}(t)\right\|_{X}: t \in T\right\}=c_{1}, \\
\sup \left\{\left\|x^{*}(t)\right\|_{X}: t \in T\right\}=c_{2},
\end{gathered}
$$

similarly (including $T_{0}=\varnothing$, Card $N<\infty$ ), for some $c_{3}, c_{4}>0$ will be

$$
\inf \left\{\left\|x^{*}(t)\right\| x: t \in T\right\}=c_{3},
$$


$\sup \left\{\left\|x_{\perp}^{*}(t)\right\| x: t \in T\right\}=c_{4}$.

Consequently, the classes of real-valued functions summable with square on $T$ on measures

$$
\begin{aligned}
& v^{*}{ }_{\perp}=\int\left\|x^{*}(\tau)\right\|_{X}^{2} \mu(d \tau), \\
& v^{*}{ }_{\perp}=\int\left\|x^{*}{ }_{\perp}(\tau)\right\|_{X}^{2} \mu(d \tau),
\end{aligned}
$$

or in other words

$$
\mathrm{L}_{2}\left(T, v^{*}, R\right)=\mathrm{L}_{2}\left(T, v^{*}{ }_{\perp}, R\right),
$$

and hence (see Theorem 2)

$$
\Omega_{E} \oplus \Omega^{*}=\Omega_{E} \oplus \Omega^{*} \text {. }
$$

If we look at Theorem 2 under foreshortening of unmanaged trajectories of a differential system (1), we can see that the analyst of output condition

$$
\mathrm{L}_{2}\left(T, v^{*}, R\right)=\mathrm{L}_{2}\left(T, v^{*}{ }_{\perp}, R\right)
$$

in the proof of Corollary 3 enables us to strengthen this theorem to the characteristic feature of elementary algebraic extension of differential realization of a finite buddle of unmanaged implementation processes

$$
N \subset A C(T, X) \times\{0\} \times\{0\} \subset \Pi .
$$

Theorem 3. In the family of free $C$-solutions the problem of singleton expansion of differential realization of the finite bundle of trajectories is solvable if and only if $T_{0}=\varnothing$.

\section{Conclusion}

The external world is too complex. And any researcher working in natural sciences is happy when he manages to understand at least some minor, trivial part of the regularities inherent in nature. The point is that in order to reach such an understanding, a researcher normally applies some simplified and idealized models (in particular, also quasi-linear ones [7]), which ignore unimportant (from his viewpoint) details, which could somehow complicate the investigation, and at the same time reflect (as he hopes) the most essential properties of the scrutinized natural object. Roughly speaking, in this process, the researcher behaves as follows: (i) the issue of choosing a class of models is solved by him within a definite branch of natural sciences; (ii) after the period of field investigations and preliminary analysis of the results, he addresses a mathematician [10, p. 14]. Mathematics allows one to draw conclusions on the basis of a class of models chosen [11].

A qualitative method of analysis of the geometric characteristics of the existence of non-stationary $\left(A, B, B^{\#}\right)_{2}$-model of vector field, including in the class of its feasible solutions advanced countable bundle of complex dynamic processes was proposed and developed in this paper. Allocated problem is mathematically self-sufficient, because it is of excep- tional interest from the standpoints of understanding the fine topological-algebraic structure of the set of controlled dynamic processes, having the differential realization (1). It is possible that the next productive intention in this study lies in the clarification (including higher-order differential realization [12-15]) of how the geometric feature (2) in the structure $\mathrm{H}_{2}$, allowing to obtain the Theorem 2 about singleton extension of dynamic bundle depends on the condition $T_{0}=\varnothing(\bmod \mu)$.

Note that the problem of elementary expansion of the differential implementation of a countable bundle of controlled multi-linear dynamic processes can also be solved on the basis of the semiadditive property $[16, p .400]$ of the non-linear functional Rayleigh -Ritz operator [17-19].

Acknowledgements: This work was supported by the Russian Foundation for Basic Research (Projects: No. 19-01-00301, №. 19-08-00746).

\section{References}

[1] V.A. Rusanov, A.V. Daneev, A.V. Lakeev and Yu.É. Linke, On the differential realization theory of non-linear dynamic processes in a Hilbert space, Far East Journal of Mathematical Sciences, Vol. 97, No. 4, 2015, pp. 495-532.

[2] Y.A. Chen, A new one-parameter inhomogeneous differential realization of the $\operatorname{spl}(2,1)$ superalgebra, International Journal of Theoretical Physics, Vol. 51, No. 12, 2012, pp. 3763-3768.

[3] V.A. Rusanov, A.V. Daneev and Yu.É. Linke, To the geometrical theory of the differential realization of dynamic processes in a Hilbert space, Cybernetics and Systems Analysis, Vol. 53, No. 4, 2017, pp. 554-564.

[4] I.V. Sergienko and V.S. Deineka, Identifying parameters of elliptic-pseudoparabolic distributed systems, Cybernetics and Systems Analysis, Vol. 47, No. 4, 2011, pp. 28-50.

[5] S.P. Novikov and I.A. Taimanov, Modern Geometric Structures and Fields, MTsNMO, Moscow, 2014 (in Russian).

[6] K. Yosida, Functional Analysis, Springer Verlag, Berlin, 1965; translated under Mir, Moscow, 1967 (in Russian).

[7] V.A. Rusanov, L.V. Antonova and A.V. Daneev, Inverse problem of non-linear systems analysis: A behavioral approach, Advances in Differential Equations and Control Processes, Vol. 10, No. 2, 2012, pp. 69-88.

[8] V.A. Rusanov, A.V. Lakeev and Yu.É. Linke, Existence of a differential realization of a dynamical system in a Banach space in the constructions of ex- 
tensions to $M_{p}$-operators, Differential Equations, Vol. 49, No. 3, 2013, pp. 346-358.

[9] J.L. Massera and J.J. Schaffer, Linear Differential Equations and Function Spaces, Academic Press, New York, 1966; translated under Mir, Moscow, 1970 (in Russian).

[10] M.D. Mesarovic and Y. Takahara, General Systems Theory: Mathematical Foundations, Academic Press, New York, 1975; translated under Mir, Moscow, 1978.

[11] J.C. Willems, System theoretic models for the analysis of physical systems, Ric. Aut., Vol. 10, 1979, pp. 71-106.

[12] A.J. van der Schaft, On realization of nonlinear systems described by higher-order differential equations, Mathematical Systems Theory Vol. 19, 1987, pp. 239-275.

[13] V.A. Rusanov, A.V. Daneev, A.V. Lakeyev and V.N. Sizykh, Higher-order differential realization of polylinear-controlled dynamic processes in a Hilbert space, Advances in Differential Equations and Control Processes, Vol. 19, No. 3, 2018, pp. 263-274.

[14] V.A. Rusanov, A.V. Banshchikov, A.V. Daneev and A.V. Lakeyev, Maximum entropy principle in the differential second-order realization of a nonstationary bilinear system, Advances in Differen- tial Equations and Control Processes, Vol. 20, No. 2, 2019, pp. 223-248.

[15] V.A. Rusanov, A.V. Daneev and Yu.É. Linke, Adjustment optimization for a model of differential realization of a multi-dimensional secondorder system, Differential Equations, Vol. 55, No. 10, 2019, pp. 1390-1396.

[16] R.E. Edwards, Functional Analysis: Theory and Applications, Holt, New York,1965; translated under Mir, Moscow, 1969.

[17] V.A. Rusanov, Algebra of sets of dynamic processes with differential realizations in a Hilbert space, Doklady Mathematics, Vol. 82, No. 1, 2010, pp. 676-677.

[18] A.V. Lakeev, Yu.É. Linke and V.A. Rusanov, On the differential realization of a secondorder bilinear system in a Hilbert space, Journal of Applied and Industrial Mathematics, Vol. 13, No. 2, 2019, pp. 261-269.

[19] A.V. Daneev, A.V. Lakeyev and V.A. Rusanov, Existence of a bilinear differential realization in the constructions of tensor product of Hilbert spaces, WSEAS Transactions on Mathematics, Vol. 19, 2020, pp. 99-107. 